Çukurova Üniversitesi Mühendislik Mimarlık Fakültesi Dergisi, 31(2), ss. 139-148, Aralık 2016

Çukurova University Journal of the Faculty of Engineering and Architecture, 31(2), pp. 139-148, December 2016

\title{
Çapraz Akışlı Saptırılmış Boru Demeti Isı Değiştiricilerinin Ekonomik Optimizasyonu
}

\author{
Mehmet Tahir ERDINÇ⿻ ${ }^{1}$, Alper YILMAZ ${ }^{* 2}$, Tuncay YILMAZ $^{1}$ \\ ${ }^{I}$ Osmaniye Korkut Ata Üniversitesi, Mühendislik Fakültesi, Makine Mühendisliği Bölümü, \\ Osmaniye, \\ ${ }^{2}$ Çukurova Üniversitesi, Mühendislik Mimarlık Fakültesi, Otomotiv Mühendislik Bölümü, \\ Adana
}

Geliş tarihi: 02.06.2016 Kabul tarihi: 23.11.2016

$\ddot{\mathbf{O} z}$

Çapraz akışlı saptırılmış boru demeti 1S1 değiştiricileri kolay temizlenebilir olmaları, hacimsel 1S1 transferinin büyük olması dolayısıyla ısı geri kazanımında ve kuru tip soğutma kulelerinde en çok tercih edilen 1 sı değiştiricilerinden biridir.

Bu çalışmada, mahal olarak Türkiye'nin 2. bölgesinden bir şehir seçilerek, 1sı değiştiricisi için ilk yatırım, işletme ve toplam giderler araştırılarak ekonomik optimizasyon yapılmıştır. İlk yatırım giderlerinde pompa, vantilatör ve 1Sı değiştiricisi fiyatları, işletme giderlerinde pompa ve vantilatörün harcadığı elektrik enerjisi dikkate alınmıştır. Ancak işletme giderleri bugünkü değere göre belirlenmiştir. Çeşitli parametrelere göre elde edilen sonuçlar grafikler şeklinde gösterilmiştir.

Anahtar Kelimeler: Çapraz akış, Saptırılmış boru demeti, Ekonomik optimizasyon

\section{Economic Optimization of Cross-flow Staggered Plain Tube Bank Heat Exchangers}

\begin{abstract}
Cross-flow staggered tube bank heat exchangers are among the most commonly used heat exchangers in heat recovery systems and closed type cooling towers due to resistance to fouling and high rate of volumetric heat transfer.

In this study, initial investment cost, operating cost and total cost for economic optimization of heat exchanger is investigated in a city of 2. climatic zone of Turkey. For initial investment cost, pump, ventilator and heat exchanger prices, and for operating cost electricity consumption of pump and ventilator are taken into consideration. But present values are used for operating costs. Results to be obtained are shown graphically for different parameters.
\end{abstract}

Keywords: Cross-flow, Staggered tube banks, Economic optimization

\footnotetext{
* Sorumlu yazar (Corresponding author): Alper YILMAZ, alpyil@cu.edu.tr
} 


\section{GíRIŞ}

Çapraz akışlı boru demeti 1Sı değiştiricileri (ÇABDID) endüstrinin birçok alanında kullanılmaktadır. Isı transferinin daha iyi olmasindan ve kolay temizlenebilme özelliklerinden dolayı saptırılmış boru demetleri tercih edilmektedir.

ÇABDID içinde 1S1 transferi kapsamlı olarak Gnielinski [1] tarafından incelenmiş ve ısı transferi için çeşitli parametreleri içeren eşitlikler verilmiştir. $\mathrm{Bu}$ 1sı değiştiricilerinde basınç kaybı için genel eşitlikler ise Gaddis [2] tarafından elde edilmiştir.

ÇABDID bilhassa kapalı yaş soğutma kulelerinde ısı değiştiricisi olarak kullanılmaktadır. Bunların modellenmesi üzerine birçok çalışma bulunmaktadır [3-6]. Ekonomik optimizasyon üzerine Caputo ve arkadaşları [7] ve Manossaldi ve arkadaşları [8] tarafından kanatçıklı borulu ısı değiştiriciler için çalışmalar yapılmıştır. $\mathrm{Bu}$ çalışmalar yapılırken en önemli fiyatlar ilk yatırım fiyatları ve işletme maliyetleri olup, bunlar hakkında geniş bilgi Taal ve arkadaşları [9] tarafından verilmiştir.

$\mathrm{Bu}$ çalışmada ÇABDID'ın ekonomik olarak optimizasyonu kanatçık içermeyen düz borular için gerçekleştirilecektir.

\section{2. ÇAPRAZ AKIŞLI SAPTIRMALI BORU DEMETI}

Çapraz akışlı saptırmalı boru demeti 1S1 değiştiricisinin sistemdeki yeri Şekil 1'de gösterilmiştir. Isı değiştiricisi dışında burada $\dot{M}_{h}$ debisini basan vantilatör ve $\dot{M}_{S}$ debisini basan pompa gösterilmiştir. Şekil 2'de ise boru demeti verilmiştir.

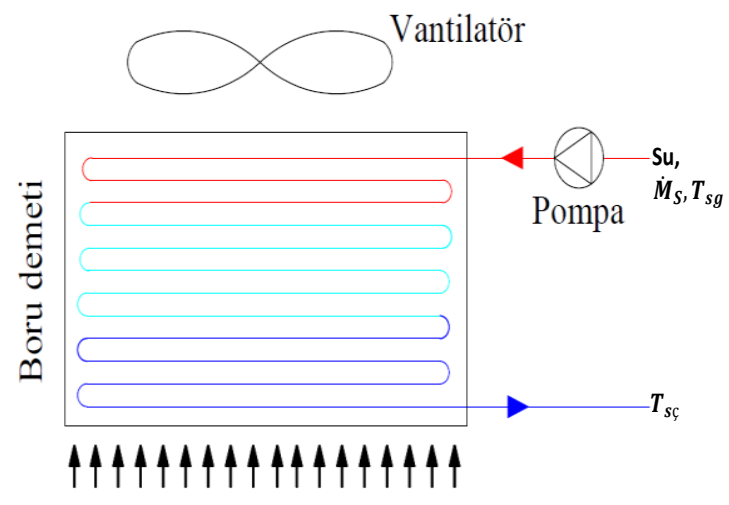

Hava $\dot{M}_{h} \boldsymbol{T}_{h g}$

Şekil 1. Isı değiştiricisinin sistemdeki yeri

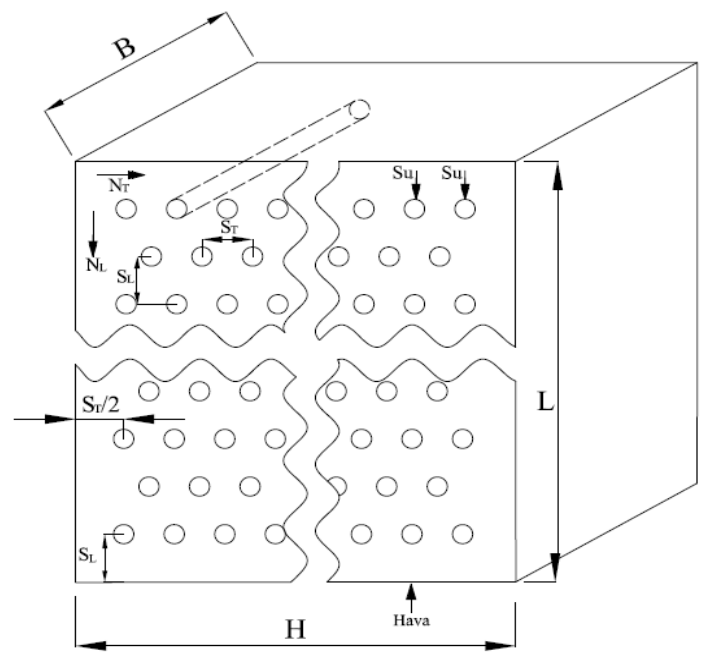

Şekil 2. Çapraz akışlı saptırmalı boru demeti

Isı değiştiricisinin borularının içinden soğutulacak su, boru dişlarından ise hava akmaktadır. Hava girişinde 1sı değiştiricisinin kesit alan boyutları B ve H'dir. Isı değiştiricisinin hava akışı doğrultusunda uzunluğu da L'dir. Boru iç ve dış çapları $d_{d}$ ve $d_{i}$ olarak verilmektedir. Şekilden de gösterildiği gibi, akışa dik yönde boru merkezleri arasındaki mesafe $s_{T}$ ve akış yönündeki mesafe ise $\mathrm{s}_{\mathrm{L}}$ 'dir. Akışa dik yönde boru sayısı $\mathrm{N}_{\mathrm{T}}$ ve akış yönünde is $\mathrm{N}_{\mathrm{L}}$ 'dir. Buna göre toplam boru sayısı 
$\mathrm{N}=2 \cdot \mathrm{N}_{\mathrm{T}} \cdot \mathrm{N}_{\mathrm{L}}$

şeklinde yazılır. Isı değiştiricisi girişindeki hava hiz1

$\mathrm{u}_{\mathrm{h}}=\frac{\dot{\mathrm{M}}_{\mathrm{h}}}{\rho_{\mathrm{h}} \cdot \mathrm{A}}$

olarak belirlenir. Burada $\mathrm{A}=\mathrm{B} . \mathrm{H}$ 1sı değiştiricisi kesit alanıdır. $\rho_{\mathrm{h}}$ hava yoğunluğu olup

$\rho_{h}=\frac{P_{h}}{R_{h} \cdot T_{h}}$

eşitliğinden hesaplanır. Burada $P_{h}$ hava basıncı, isı değiştiricisinin bulunduğu mahaldeki rakım $\mathrm{H}_{\mathrm{ma}}$ verildiğinde ASHRAE [10] tarafindan verilen bağıntıdan hesaplanır:

$\mathrm{P}_{\mathrm{h}}=101,325 \cdot\left(1-2,256 \cdot \frac{\mathrm{H}_{\mathrm{ma}}}{100.000}\right)^{5,256}$

Burada $P_{h} \quad \mathrm{kPa} \quad$ ve $\quad H_{m a} \quad$ da $\quad \mathrm{m}$ olarak birimlendirilmiştir.

Isı değiştiricisi eni $\mathrm{B}$ ile $1 \mathrm{~S} 1$ değiş̧tiricisi içindeki tek boru uzunluğu $B_{L}$ Eşitlik 3' te gösterilmiş olup, aralarındaki ilişki aşağıda verilmiştir.

$\mathrm{B}_{\mathrm{L}}=\mathrm{B}+(\pi-2) \mathrm{s}_{\mathrm{L}}$

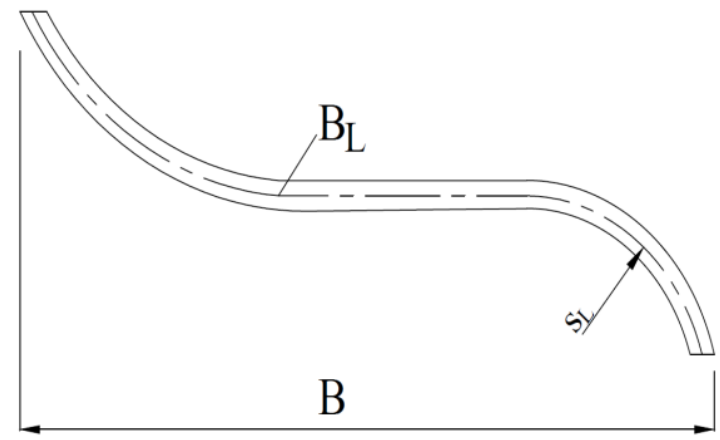

Şekil 3. $B$ ve $B_{L}$ 'nin gösterimi

\section{ISI TRANSFERİ VE BASINÇ KAYBININ HESAPLANMASI}

$\dot{M}_{s 1}$ ve $\dot{V}_{s 1}$ her kolondan akan suyun kütlesel ve hacimsel debileri olup, aşağıda verilmiştir:

$\dot{\mathrm{M}}_{\mathrm{s} 1}=\frac{\dot{\mathrm{M}}_{\mathrm{s}}}{2 \mathrm{~N}_{\mathrm{T}}}$

$\dot{\mathrm{V}}_{\mathrm{s} 1}=\frac{\dot{\mathrm{M}}_{\mathrm{s} 1}}{\rho_{\mathrm{s}}}$

Su kütlesel debisi ise transfer edilmek istenen $\dot{Q}$ isısından belirlenir:

$\dot{\mathrm{M}}_{\mathrm{s}}=\frac{\dot{\mathrm{Q}}}{\mathrm{c}_{\mathrm{ps}}\left(\mathrm{T}_{\mathrm{sg}}-\mathrm{T}_{\mathrm{sc}}\right)}$

Burada $T_{s g}$ ve $T_{s c ̧}$ suyun giriş ve çıkış sıcaklıkları olup, $\mathrm{c}_{\mathrm{ps}}$ ' de suyun özgül 1sısıdır. Boru içindeki su hiz1

$\mathrm{u}_{\mathrm{S}}=\frac{\dot{\mathrm{V}}_{\mathrm{s} 1}}{\mathrm{~A}_{\mathrm{i}}}$

Bağıntısından ve boru içi kesit alanı da

$\mathrm{A}_{\mathrm{i}}=\frac{\pi}{4} \mathrm{~d}_{\mathrm{i}}^{2}$

eşitliğinden bulunmalıdır.

Hesaplarda gerekli olacak $\operatorname{Re}_{\mathrm{s}}$ sayıs 1

$\operatorname{Re}_{\mathrm{s}}=\frac{\mathrm{u}_{\mathrm{s}} \mathrm{d}_{\mathrm{i}}}{\mathrm{v}_{\mathrm{s}}}$

şeklinde hesaplanır. Kinematik viskosite $v_{s}$ dahil, fiziksel özellikler suyun ortalama sıcaklı̆̆ $1 T_{s m}$ 'de alınmalıdır.

$\mathrm{T}_{\mathrm{sm}}=\frac{\mathrm{T}_{\mathrm{sg}}+\mathrm{T}_{\mathrm{sc}}}{2}$ 
Boru içinden akan suyun basınç kaybı

$\Delta \mathrm{P}_{\mathrm{s}}=\left(\zeta_{\mathrm{y}}+\zeta_{\mathrm{s}} \cdot \frac{\mathrm{L}_{\mathrm{B} 1}}{\mathrm{~d}_{\mathrm{i}}}\right) \cdot \frac{\rho_{\mathrm{s}} \mathrm{u}_{\mathrm{s}}^{2}}{2}$

şeklinde belirlenir. $L_{B 1}$ uzunluğu suyun bir kolonda akma uzunluğu olup

$\mathrm{L}_{\mathrm{B} 1}=\mathrm{N}_{\mathrm{L}} \cdot \mathrm{B}_{\mathrm{L}}$

eşitliğinden bulunur.

Su tarafindaki 1sı transferi katsayısı

$\mathrm{h}_{\mathrm{s}}=\mathrm{Nu}_{\mathrm{s}} \cdot \frac{\mathrm{k}_{\mathrm{s}}}{\mathrm{d}_{\mathrm{i}}}$

şeklinde hesaplanır. Burada $k_{s}$ suyun 1s1 iletim katsayısıdır. Yerel basınç kayıp katsayısı giriş ve çıkış kayıpları dikkate alınarak $\zeta_{y}=2$ alınacaktır. Sürtünme kayıp katsayısı ve Nusselt sayısı laminar ve türbülanslı akış durumları için aşağıda verilen eşitliklerden bulunabilir [11].

$\operatorname{Re}_{i} \leq 2300 \quad$ için

$$
\begin{aligned}
& N u_{s}=3,66+\frac{0,0677 z_{s}^{-1,33}}{1+0,1 \operatorname{Pr}_{s}^{0,117} z_{s}^{-0,83}} \\
& z_{s}=\frac{L_{B 1}}{d_{i}} \frac{1}{\operatorname{Re}_{s} \operatorname{Pr}_{s}} \\
& \xi_{s}=\frac{64}{\operatorname{Re}_{s}}+1,33
\end{aligned}
$$

$\operatorname{Re}_{s}>2300$ için

$$
\mathrm{Nu}_{\mathrm{s}}=\frac{\lambda_{\mathrm{s}}}{8} \frac{\left(\operatorname{Re}_{\mathrm{s}}-1000\right) \operatorname{Pr}_{\mathrm{s}}}{1+12,7\left(\lambda_{\mathrm{s}} / 8\right)^{1 / 2}\left(\operatorname{Pr}_{\mathrm{s}}^{2 / 3}-1\right)}
$$

$$
\lambda_{s}=\frac{1}{\left(1,82 \log \operatorname{Re}_{s}-1,64\right)^{2}}
$$

$$
\frac{1}{\sqrt{\xi_{s}}}=3,48-1,7372 \ln \left(\frac{\varepsilon}{d_{i} / 2}+\frac{9,35}{\operatorname{Re}_{s} \sqrt{\xi_{s}}}\right)
$$

Burada $\varepsilon$ iç borudaki bağıl pürüzlülük

$$
\varepsilon=\frac{s_{p}}{d_{i}}
$$

olup sp'de mutlak pürüzlülüktür.

Hava ile boru dışı arasındaki ısı transferi katsayısı

$$
\mathrm{h}_{\mathrm{h}}=\mathrm{Nu}_{\mathrm{h}} \cdot \frac{\mathrm{k}_{\mathrm{h}}}{\mathrm{d}_{\mathrm{i}}}
$$

bağıntısından bulunur. $k_{h}$ havanın 1sı iletim katsayısıdır. $h_{h}$ Gnielinski [1] tarafindan açıklanan yönteme göre hesaplanacaktır. Bu metoda göre

$$
N u_{L}=0,3+\left(N u_{L, l}^{2}+N u_{L, t}^{2}\right)^{0,5}
$$

$$
N u_{L, l}=0,664 \operatorname{Re}_{V, L}^{0,5} \operatorname{Pr}_{h}^{1 / 3}
$$

$N u_{L, t}=\frac{0,037 \operatorname{Re}_{V, L}^{0,8} \operatorname{Pr}_{h}}{1+2,443 \operatorname{Re}_{V, L}^{-0,1}\left(\operatorname{Pr}_{h}^{2 / 3}-1\right)}$

$$
N u_{h, \infty}=\frac{2}{\pi} N u_{L}
$$

$$
N u_{N}=f_{A, N} N u_{a}
$$

$$
f_{A, N}=\frac{1+\left(N_{L}-1\right) f_{A}}{N_{L}}
$$

$$
f_{A}=1+\frac{2}{3 s_{L}^{*}}
$$

eşitlikleriyle elde edilir. 
Burada $\operatorname{Re}_{V, L}$ değeri

$$
\begin{aligned}
& \operatorname{Re}_{V, L}=\frac{\pi}{2} \frac{\left(1-\frac{1}{s_{T}^{*}}\right)}{\left(1-\frac{\pi}{4 s_{T}^{*}}\right)} \operatorname{Re}_{\text {max }} \\
& \operatorname{Re}_{\text {max }}=\frac{u_{\text {max }} d_{d}}{v} \\
& N u_{h}=\frac{2}{\pi} N u_{L}
\end{aligned}
$$

bağıntıları ile belirlenir. Burada ayrıca

$$
\begin{gathered}
s_{L}^{*}=\frac{s_{L}}{d_{d}} \\
s_{T}^{*}=\frac{s_{T}}{d_{d}}
\end{gathered}
$$

şeklinde tarif edilmişlerdir.

Isı değiştiricisinde transfer edilen 1s1

$\dot{\mathrm{Q}}=\mathrm{U} \cdot \mathrm{F} \cdot \Delta \mathrm{T}_{\mathrm{m}}$

olarak belirlenir. Su giriş ve çıkış sıcaklıkları ile hava giriş sıcaklığı verildiğinde, hava çıkış sıcaklığı bellidir. Bu durumda $\Delta T_{m}$ ve UF değerleri

$$
\begin{aligned}
& \Delta \mathrm{T}_{\mathrm{m}}= \frac{\left(\mathrm{T}_{\mathrm{sg}}-\mathrm{T}_{\mathrm{hc}}\right)-\left(\mathrm{T}_{\mathrm{sc}}-\mathrm{T}_{\mathrm{hg}}\right)}{\ln \left(\frac{\left(\mathrm{T}_{\mathrm{sg}}-\mathrm{T}_{\mathrm{hc}}\right)}{\left(\mathrm{T}_{\mathrm{sc}}-\mathrm{T}_{\mathrm{hg}}\right)}\right)} \\
& \frac{1}{U F}=\frac{1}{\frac{1}{h_{i} F_{i}}+\frac{s_{d}}{k_{d} F_{d m}}++\frac{1}{h_{h} F_{d}}}
\end{aligned}
$$

şeklinde bulunurlar. Ayrıca boru iç, dış ve ortalama yüzey alanları
$\mathrm{F}_{\mathrm{d}}=\mathrm{N} \cdot \pi \cdot \mathrm{d}_{\mathrm{d}} \cdot \mathrm{B}_{\mathrm{L}}$

$\mathrm{F}_{\mathrm{i}}=\mathrm{N} \cdot \pi \cdot \mathrm{d}_{\mathrm{i}} \cdot \mathrm{B}_{\mathrm{L}}$

$F_{m}=\frac{F_{d}-F_{i}}{\ln \frac{F_{d}}{F_{i}}}$

olarak belirlenir. $\mathrm{u}_{\max }$ ile $\mathrm{u}_{\mathrm{h}}$ arasında

$u_{\max }=\frac{s_{T}^{*}}{2\left(s_{d}^{*}-1\right)} u_{h}$

$s_{d}^{*}=\left[s_{L}^{* 2}+\left(s_{L}^{*} / 2\right)^{2}\right]^{0,5}$

bağıntıları geçerlidir. Hava tarafındaki basınç kayb1

$\Delta P_{h}=\lambda_{N} N_{L} \frac{\rho_{h} u_{\max }^{2}}{2}$

bağıntısıyla hesaplanır. $\lambda_{N}$ değeri de Gaddis [2] tarafından verilen eşitlikle elde edilir.

$\lambda_{h}=\lambda_{h l}+\lambda_{h t}\left[1-\exp \left(-\frac{\operatorname{Re}_{\text {max }}+200}{1000}\right)\right]$

$s_{L}^{*} \geq 0,5\left(2 s_{T}^{*}+1\right)^{0,5}$ için

$\lambda_{h l}=\frac{280 \pi\left[\left(s_{L}^{* 0,5}-0,6\right)^{2}+0,75\right]}{\operatorname{Re}_{\max }\left(4 s_{L}^{* 2} s_{T}^{*}-\pi\right) s_{T}^{* 1,6}}$

$s_{L}^{*}<0,5\left(2 s_{T}^{*}+1\right)^{0,5}$ için ise

$\lambda_{h l}=\frac{280 \pi\left[\left(s_{L}^{* 0,5}-0,6\right)^{2}+0,75\right]}{\operatorname{Re}_{\text {max }}\left(4 s_{L}^{* 2} s_{T}^{*}-\pi\right) c^{* 1,6}}$ 
$c^{*}=\left(s_{L}^{* 2}+s_{T}^{* 2} / 4\right)^{0,5}$

yazılır. $\lambda_{h t}$ ise aşağıdaki bağıntıdan hesaplanır.

$\lambda_{h t}=\left[2,5+\frac{1,2}{\left(s_{T}^{*}-0,85\right)^{1,08}}+0,4\left(\frac{s_{L}^{*}}{s_{T}^{*}}-1\right)^{3}-0,01\left(\frac{s_{T}^{*}}{s_{L}^{*}}-1\right)^{3}\right] / \mathrm{Re}_{\text {max }}^{0,25}$

\section{4. İSTETME GIDERLERINIIN HESABI}

İşletme giderleri olarak vantilatör ve pompanın harcadıkları elektrik enerjisi dikkate alınacaktır. Su pompasının işletme gideri

$\dot{\mathrm{W}}_{\mathrm{s}}=\frac{\Delta \mathrm{P}_{\mathrm{s}} \cdot \dot{\mathrm{V}}_{\mathrm{s}}}{\eta_{\mathrm{pT}}}$

şeklinde hesaplanır. $\eta_{p T}$ toplam pompa verimi olup; pompa, aktarma organı ve elektrik motoru verimlerini içermektedir.

Vantilatör toplam basınç kaybı

$\Delta \mathrm{P}_{\mathrm{V}}=\Delta \mathrm{P}_{\mathrm{h}}+\xi_{\mathrm{h}} \frac{\rho \mathrm{u}_{\mathrm{h}}^{2}}{2}$

olarak belirlenir. $\xi_{h}$ yerel basınç katsayısı olup, giriş ve çıkış kayıpları dikkate alınarak $\xi_{h}=2$ olarak kabul edilmiştir. Vantilatörün harcadığı güç de

$\dot{\mathrm{W}}_{\mathrm{V}}=\frac{\Delta \mathrm{P}_{\mathrm{V}} \cdot \dot{\mathrm{V}}_{\mathrm{h}}}{\eta_{\mathrm{VT}}}$

şeklinde hesaplanmıştır. $\eta_{V T}$ toplam vantilatör verimi olup; vantilatör, aktarma organı ve elektrik motoru verimlerini içermektedir. Toplam elektrik tüketimi
$\dot{\mathrm{W}}=\dot{\mathrm{W}}_{\mathrm{V}}+\dot{\mathrm{W}}_{\mathrm{p}}$

şeklinde belirlenir. Yıllık harcanan elektrik tutarı FI'da

$\mathrm{FI}=\dot{\mathrm{W}} \cdot \mathrm{YS} \cdot \mathrm{fe} / 1000$

olarak elde edilir. YS yıllık çalışma saati olup, genelde $Y S=8000$ olarak kabul edilmektedir. fe ise elektrik birim fiyatı olup, 2016 ilk yarıyılda $\mathrm{fe}=0,2 \mathrm{TL} / \mathrm{kWh}$ olarak alınabilir.

Ancak, ilk yatırım masraflarıyla karşılaştırmak için işletme masraflarının bugünkü değeri $\mathrm{FI}_{\mathrm{BD}}$ 'nin bulunması gerekmektedir. $\mathrm{FI}_{\mathrm{BD}}$ 'de

$\mathrm{FI}_{\mathrm{BD}}=\mathrm{FI} \cdot \mathrm{F}_{\mathrm{BD}}$

$\mathrm{F}_{\mathrm{BD}}=\frac{\left(1+\mathrm{i}_{\mathrm{r}}\right)^{\mathrm{n}}-1}{\mathrm{i}_{\mathrm{r}} \cdot\left(1+\mathrm{i}_{\mathrm{r}}\right)^{\mathrm{n}}}$

olarak elde edilir [12]. $\mathrm{i}_{\mathrm{r}}$ reel faiz olup, enflasyon $\mathrm{i}_{\mathrm{e}}$ ve nominal faiz $i_{n}$ den elde edilir [12]:

$\mathrm{i}_{\mathrm{r}}=\frac{\mathrm{i}+\mathrm{i}_{\mathrm{n}}}{1+\mathrm{i}_{\mathrm{e}}}-1$

Eşitlik 56'da n pompa ve vantilatör amortizasyon yılidir.

\section{ILK YATIRIM GIDERLERININ HESABI}

İlk yatırım fiyatı FY

$\mathrm{FY}=\mathrm{FYV}+\mathrm{FYP}+\mathrm{FYE}$ 
olarak, vantilatör, pompa ve 1S1 değiştiricisi ilk yatırım fiyatlarından oluşur. Burada ısı değiştiricisi fiyat1

$\mathrm{FYE}=\mathrm{L}_{\mathrm{B}} \cdot \mathrm{f}_{\mathrm{B}} \cdot \mathrm{f}_{\mathrm{E}}$

şeklinde bulunabilir. Burada da $\mathrm{L}_{\mathrm{B}}$ 1S1 değiştiricisi boru uzunluğu olup

$\mathrm{L}_{\mathrm{B}}=\mathrm{B}_{\mathrm{L}} \cdot \mathrm{N}$

olarak hesaplanır. $f_{B}$ birim boru uzunluğu, $f_{E}$ ' de imalat katsayısıdır. Vantilatör fiyatı FYV, 2015 y1lı birim fiyat listesine göre [13] Şekil 4'te verilmiştir. Bu değerler eşitlik olarak

$F Y V=2,97 \cdot \dot{V}_{h}^{0,64}$

şeklinde bulunmuştur. FYV, TL olarak ve $\dot{V}_{h}$ 'de $\mathrm{m}^{3} / \mathrm{h}$ olarak birimlendirilmiştir.

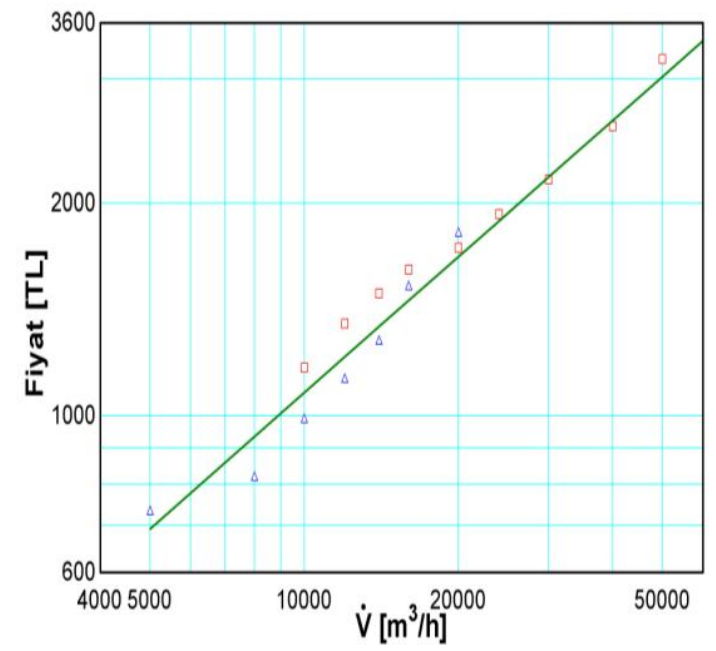

Şekil 4. FYV' nin debi ile değişimi

Pompa fiyatı FYP yine birim fiyat listesine göre

[14] Şekil 5'te gösterilmiştir. FYP debi ve basınca bağlı olarak aşağıdaki gibi bulunmuştur.

$\mathrm{PYP}=255 \cdot \mathrm{P}_{\mathrm{p}}^{0,42} \cdot \dot{\mathrm{V}}_{\mathrm{p}}^{0,5}$

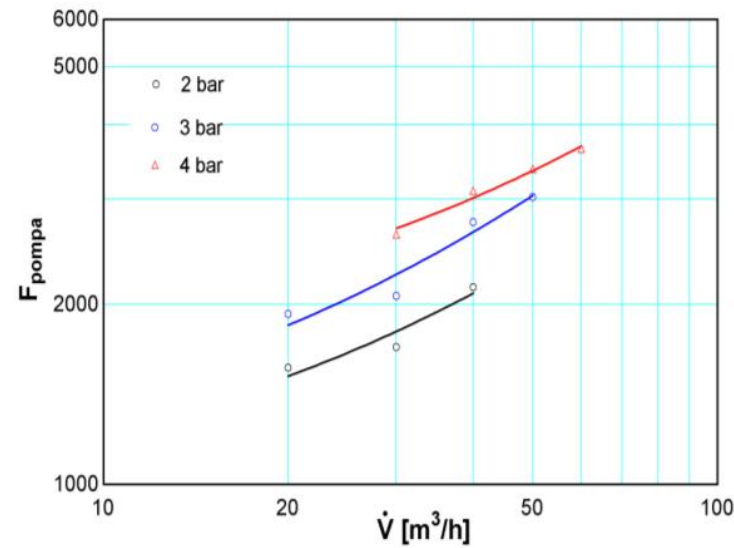

Şekil 5. FYP'nin debi ve basınç ile değişimi

Pompa çıkış basıncı P bar ve pompa debisi $\dot{V}_{p}$ 'de $m^{3} / h$ olarak verilmektedir. PYP'de TL olarak bulunmaktadır. Toplam fiyat da

$\mathrm{FYI}_{\mathrm{BD}}=\mathrm{FY}+\mathrm{FI}_{\mathrm{BD}}$

olarak hesaplanır.

\section{SONUÇLAR}

Hesaplarda 1sı değiştiricilerde en çok kullanılan 3/4"'lik çelik boru alınacak olup, $d_{d}=26,9 \mathrm{~mm}$, $\mathrm{d}_{\mathrm{i}}=21,1 \mathrm{~mm}$ 'dir. Mahal olarak Türkiye'de 2. Bölge seçilmiş olup, kış aylarına uygun ortalama dış sıcaklık $T_{h g}=1^{\circ} \mathrm{C}$ alınmıştır. 2. Bölgede şehir olarak Gaziantep seçilmiş olup, $\mathrm{H}_{\mathrm{ma}}=840$ m'dir. Borular eşkenar üçgen şeklinde yerleştirilmiş olup, bundan dolayı

$\mathrm{S}_{\mathrm{L}}=\frac{\sqrt{3}}{2} \mathrm{~s}_{\mathrm{T}}$

olarak alınacaktır. Isı değiştiricisi kesit alanı kare olarak kabul edilecek ve bundan dolayı

$\mathrm{B}=\mathrm{H}$

olarak hesaplar yapılacaktır. Hesaplanan 1S1 değiştiricisinin yoğuşturucu soğutulması için 
kullanıldığı düşünülürse, su giriş ve çıkış sıcaklıkları Gaziantep için $T_{s g}=33^{\circ} \mathrm{C}$ ve $T_{s c ̧}=$ $28^{\circ} \mathrm{C}$ olarak kabul edilecektir. Kullanılan borunun birim fiyatı 2016 fiyatlariyla $f_{B}=5,06 \mathrm{TL} / \mathrm{m}$ ve $\mathrm{f}_{\mathrm{E}}=1,5$ kabul edilmiştir. Ayrıca nominal faiz $i_{n}=0,11$ ve enflasyon da $i_{e}=0,07$ olarak alınacaktır. Pompa çıkış basıncının da $\mathrm{P}_{\mathrm{p}}=2$ bar olduğu düşünülmektedir.

Yukarıda verilen değerler dikkate alınarak ekonomik optimizasyon için değişkenler olarak transfer edilen 1sı $\dot{Q}$, hava kütle debisi $\dot{M}_{h}$, ve akışa dik yönde borular arası mesafe $\mathrm{s}_{\mathrm{T}}$ alınacaktır.

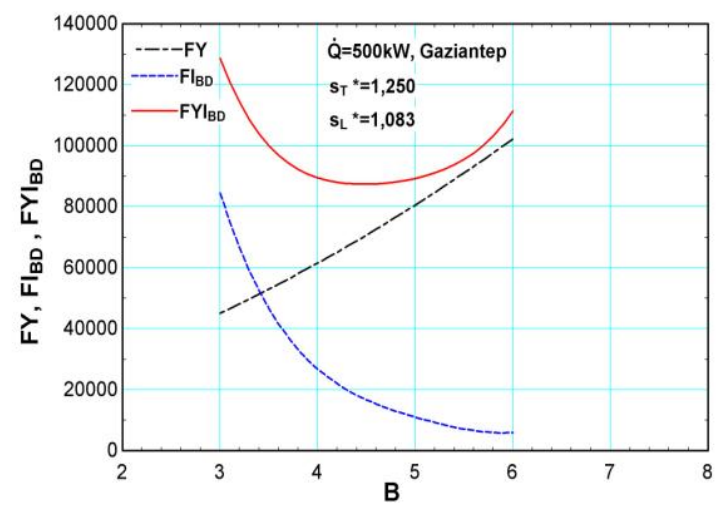

Şekil 6. İlk yatırım işletme bugünkü değer ve toplam bugünkü fiyatın $\mathrm{B}$ ile değişimi

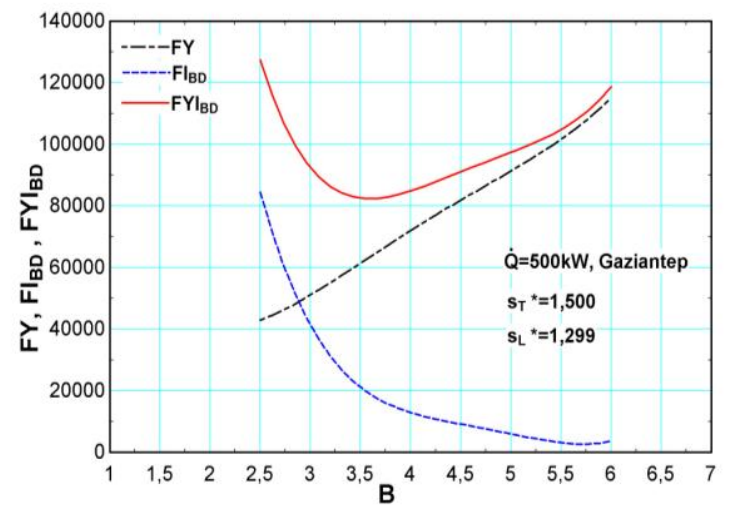

Şekil 7. İlk yatırım işletme bugünkü değer ve toplam bugünkü fiyatın $\mathrm{B}$ ile değişimi

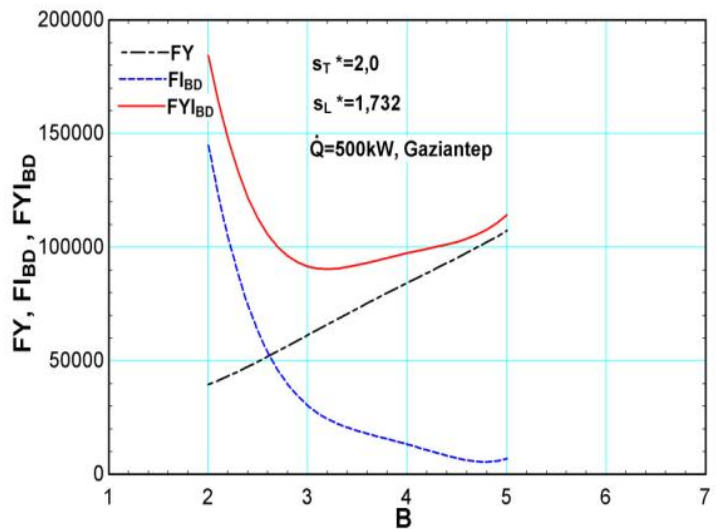

Sekil 8. İlk yatırım işletme bugünkü değer ve toplam bugünkü fiyatın $B$ ile değişimi

Şekil 6, 7 ve 8'de 1s1 değiştiricisi, pompa ve vantilatörün ilk yatırım tutarı FY ile işletme masrafları bugünkü değeri ve toplam masraf bugünkü değer $\mathrm{FI}_{\mathrm{BD}}, \mathrm{FYI}_{\mathrm{BD}}$ 1S1 değiştiricisi eni B'nin fonksiyonu olarak verilmiştir. İlk yatırım masrafları B ile artarken işletme masrafları da azalmaktadır. Ancak belirli bir B değerinde toplam masraf en küçük değere erişmekte olup, bu değer 1S1 değiştiricisinin optimum ekonomik değeridir. $\mathrm{Bu}$ üç diyagramda $s_{T}^{*}$ değeri sırasılyla $1,25,1,50$ ve 2,00 olarak alınmıştır. Optimum 1sı değiştiricisi eni $B_{\text {opt }}$ ve optimum toplam bugünkü fiyat da $F_{\text {opt }}$ olarak gösterilirse, bunlar $\mathrm{s}_{\mathrm{T}, \mathrm{o}}^{*}$ 'un fonksiyonu olarak Şekil 9 ve 10 verilmiştir.

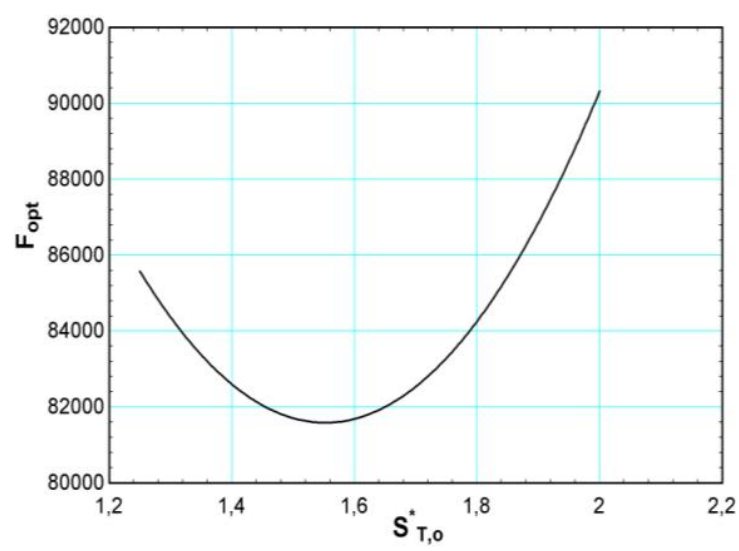

Şekil 9. $\mathrm{F}_{\text {opt }}$ değerinin $s_{T, o}^{*}$ ile değişimi 


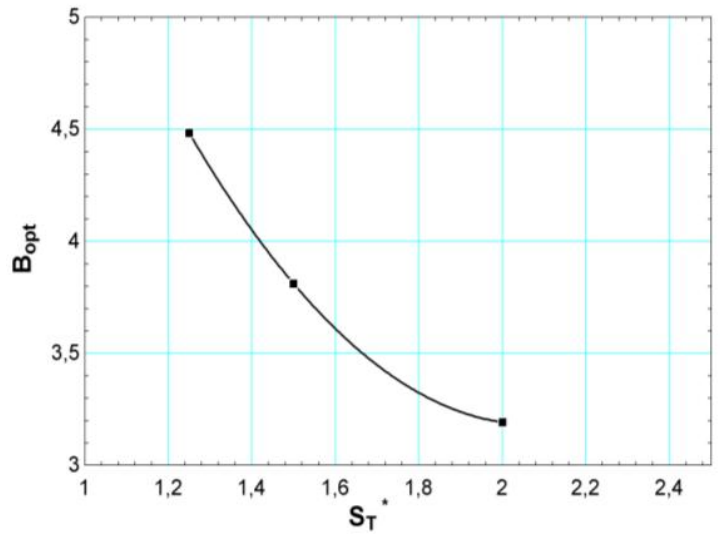

Şekil 10. $B_{\text {opt }}$ değerinin $s_{T, o}^{*}$ ile değişimi

Şekil 9'dan görüleceği gibi $\mathrm{F}_{\text {opt }}$ değeri $s_{T, o}^{*}$ ile önce artmakta olup, yaklaşık $s_{T, o}^{*}=1,55$ 'te en küçük olmaktadır. Buna karşı $\mathrm{B}_{\text {opt }}$ değeri $s_{T, o}^{*}$ ile düşmektedir. Bunun nedeni $S_{T, o}^{*}$ küçüldüğünde basınç kayıpları hızla artmakta, bu artışı telafi etmek için ise $\mathrm{B}_{\mathrm{opt}}$ düşmektedir.

Şekil 11 ve 12 'de ise $s_{T}^{*}=1,5$ durumunda $\dot{Q}_{e}$ değeri, Şekil 7'de verilenin yarısına ve iki katına çıkarılmıştır. Bu durumlarda hava kütle debisi de yarıya ve iki katına çıkarılmıştır. $B_{\text {opt }}$ ve $F_{\text {opt }}$ değerleri beklendiği gibi kule yükü $\dot{\mathrm{Q}}_{\mathrm{k}}$ ile artmaktadır. Şekil 13 ve 14'te $F_{\text {opt }}$ ve $B_{\text {opt }}$ değerlerinin $\dot{Q}_{e}$ ile değişimi verilmiş olup, hem $\mathrm{F}_{\mathrm{opt}}$ hem de $\mathrm{B}_{\mathrm{opt}}, \dot{Q}_{e}$ ile artmaktadır.

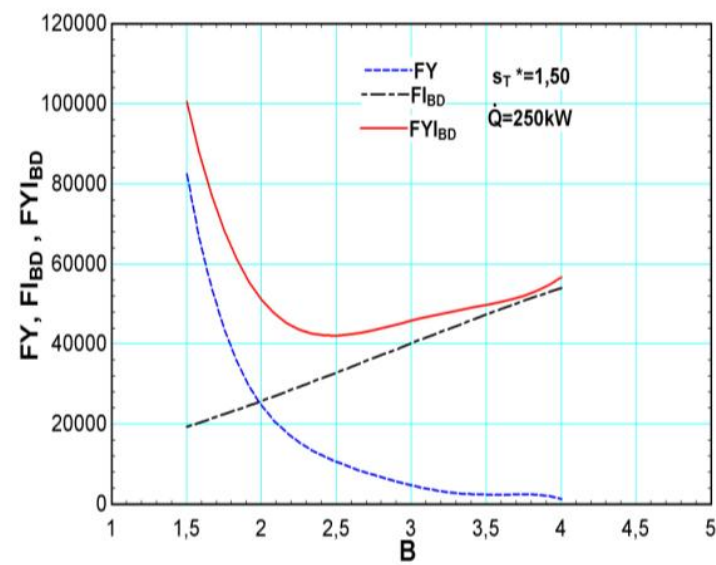

Şekil 11. Fiyatların B ile değişsimi

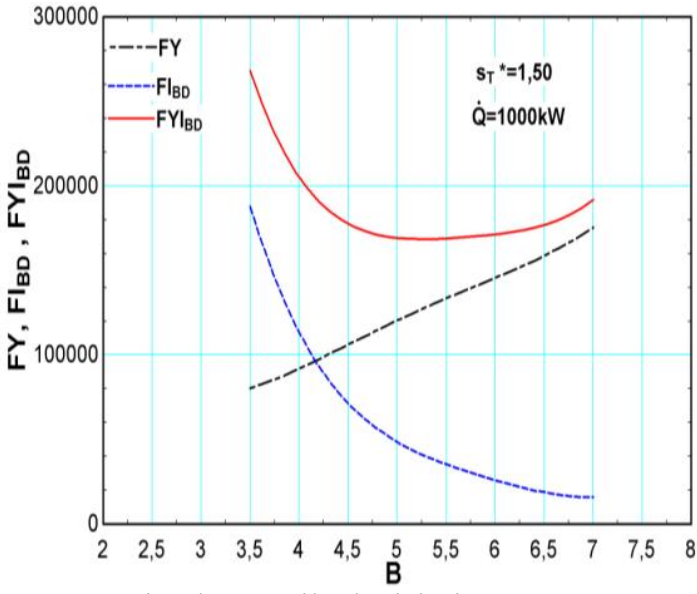

Şekil 12. Fiyatların B ile değişimi

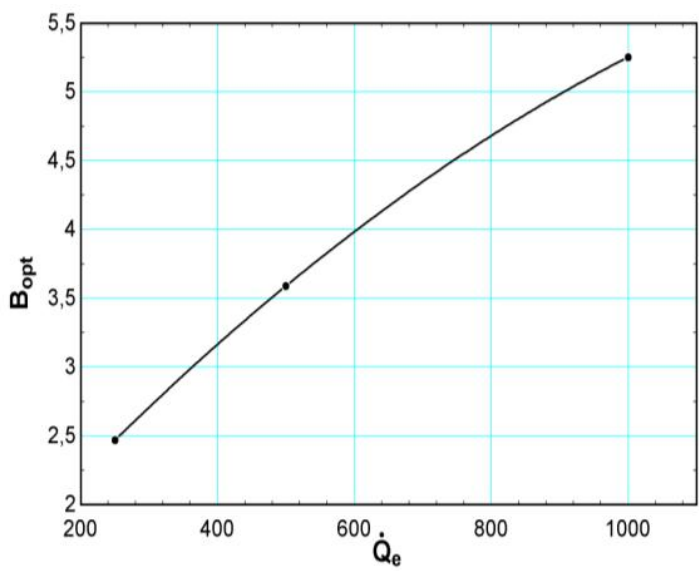

Şekil 13. $\mathrm{B}_{\mathrm{opt}}$ değerinin $\dot{Q}_{e}$ ile değişimi

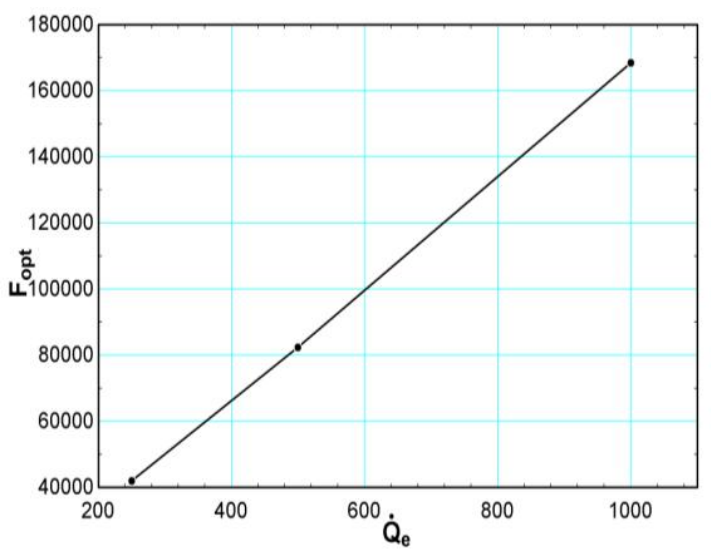

Şekil 14. $\mathrm{F}_{\mathrm{opt}}$ değerinin $\dot{Q}_{e}$ ile değişimi 


\section{KAYNAKLAR}

1. Gnielinski, V., 2010. Heat Transfer in Cross Flow Around Single Rows of Tubes and Through Tube Bundles, in VDI Heat Atlas, ed. VDI, Springer Verlag, Berlin, Germany, pp. 725-729.

2. Gaddis, E.S., 2010. Pressure Drop of Tube Bundles in Cross Flow, in VDI Heat Atlas, ed. VDI, Springer Verlag, Berlin, Germany, pp. 1076-1091.

3. Yılmaz, A., Erdinç, M. T., 2016. A New Model for the Calculation of Closed Wet Cooling Towers, International Conference on Natural Science and Engineering (ICNASE'16), March 19-20,p. 2454-2471, Kilis.

4. Zalewski, W., Niezgoda-Zelasko, B., Litwin, M., 2000. Optimization of Evaporative Fluid Coolers, International Journal of Refrigeration, 23, 553-565.

5. Gan, G., Riffat, S.B., 1999. Numerical Simulation of Closed Wet Cooling Towers for Chilled Ceiling Systems, Applied Thermal Engineering, 19, 1279-1296.

6. Hasan A., Siren K., 2001. Theoretical and Computational Analysis of Closed Wet Cooling Towers and its Applications in Cooling of Buildings, Energy and Building, 34,477-486

7. Caputo A. C., Pelagage P. M., Salin, P., 2008. Heat Exchanger Design Based on Economic Optimization, Applied Thermal Engineering, 28, 1151-1159.

8. Nabassaldi J.I., Nicolas J.S., Mussati S.F., 2014. Optimization Mathematical Model for the Detailed Design of Air Cooled Heat Exchangers, Energy, 64, 734-746.

9. Taal, M., Bulatov I., Klemes J., Stehlik P., 2003. Cost Estimation and Energy Price Forecasts for Economic Evaluation of Retrofit Projects, Applied Thermal Engineering, 23, 1819-1835.

10. ASHRAE Handbook Fundamentals, 2009. Chapter 1: Psychometrics, Atlanta, Ga.: American Society of Heating Refrigerating and Air-Conditioning Engineers.

11. Yılmaz, T., 1999. Teorik ve Uygulamalı Is1 Transferi, Papatya Yayıncılık.
12. Mishra, S.. 2010. Engineering Economics and Costing, PHI Learning Private Limited.

13. Çevre ve Şehircilik Bakanlığ Fiyat Listesi, Poz No: 253.101-253.107253.201-253.209.

14. Çevre ve Şehircilik Bakanlığ Fiyat Listesi, Poz No:217.122-217.160. 